\title{
PRIMITIVE RECURSIVE FUNCTIONS. II
}

\author{
RAPHAEL M. ROBINSON
}

In this note, we shall prove two theorems which are modifications of a result of PRF. ${ }^{1}$ The second of these is used by the author in another paper. ${ }^{2}$

In PRF, we made much use of pairing functions, that is, of functions $J(u, v), K x, L x$ such that

$$
K J(u, v)=u, \quad L J(u, v)=v,
$$

but no fixed choice of pairing functions was made. In this note, we shall use only the Cantor pairing functions. Here

$$
2 J(u, v)=(u+v)^{2}+3 u+v
$$

In this case, the formula $x=J(u, v)$ establishes a one-to-one correspondence between pairs of natural numbers $(u, v)$ and all natural numbers $x . K$ and $L$ are defined as the inverse functions. Notice that $K x$ is the excess of $x$ over a triangular number. In addition to the previous formulas, we also have $J(K x, L x)=x$.

THEOREM 1. All primitive recursive functions of one variable can be obtained by starting with the two functions $S$ and $K$ (or with $S$ and $L$ ), and repeatedly using any of the formulas

$$
F x=A x+B x, \quad F x=B A x, \quad F x=B^{x} 0
$$

to construct a new function from known functions $A$ and $B$.

Proof. Here $S$ is the successor function, and the formula $F x=B^{x} 0$ means that $F 0=0$ and $F S x=B F x$. According to PRF, $\S 7$, Theorem 3 , a similar result is valid in which we take $S$ and $E$ as initial functions, where $E x=x-\left[x^{1 / 2}\right]^{2}$. Thus to prove the theorem, we need only show that $E$ can be defined.

Suppose first that the initial functions are $S$ and $K$. We start by defining the functions

$$
I x=S^{x} 0, \quad O x=I^{x} 0, \quad \operatorname{sgn} x=(S O)^{x} 0 .
$$

Presented to the Society, November 27, 1954; received by the editors October 2, 1954.

${ }^{1}$ R. M. Robinson, Primitive recursive functions, Bull. Amer. Math. Soc. vol. 53 (1947) pp. 925-942.

2 R. M. Robinson, Arithmetical representation of recursively enumerable sets, to appear. 
Then $I x=x, O x=0, \operatorname{sgn} 0=0, \operatorname{sgn} x=1$ for $x>0$.

It is easily seen that

$$
x(x+3) / 2=B^{x} 0 \quad \text { with } B y=y+K y+2 .
$$

Using the fact that

$(x+y)(x+y+3) / 2+2 x+3=(x+y+2)(x+y+3) / 2+x-y$, we see that

$$
K[(x+y)(x+y+3) / 2+2 x+3]=x-y \quad \text { for } x \geqq y .
$$

This enables us to compute differences. In particular, we can put

$$
x^{2}=2[x(x+3) / 2]-3 x .
$$

We shall now show how to define the function

$$
F x=2 x-\left[x^{1 / 2}\right] .
$$

Notice that $F\left(n^{2}\right)=n(2 n-1)$ and $F(n(n+1))=n(2 n+1)$. Thus every triangular number is of the form $F x$. Since $F x$ is an increasing function of $x$, we see that $F x$ is triangular if and only if $x$ is of the form $n^{2}$ or $n(n+1)$. Also, $S F x$ can be triangular only if $S F x=F S x$. Now

$$
F S x=\left\{\begin{array}{l}
S F x \text { if } S x \text { is a square, } \\
S S F x \text { if } S x \text { is not a square. }
\end{array}\right.
$$

Hence $S F x$ is triangular if and only if $S x$ is square. Consequently,

$$
F S x=S F x+\operatorname{sgn} K S F x .
$$

In other words,

$$
F x=B^{x} 0 \quad \text { with } B y=S y+\operatorname{sgn} K S y .
$$

We can now define $E$ by the equations

$$
\begin{aligned}
& {\left[x^{1 / 2}\right]=2 x-F x,} \\
& E x=x-\left[x^{1 / 2}\right]^{2} .
\end{aligned}
$$

This completes the proof for the case where the initial functions are $S$ and $K$. If the initial functions are $S$ and $L$, we may first define $K$ by the formula

$$
K x=L(x+2 L x+2),
$$

which is easily verified.

THEOREM 2. All primitive recursive functions of one variable can be 
obtained by starting with the two functions $S$ and $K$ (or with $S$ and $L$ ), and repeatedly using any of the formulas

$$
F x=J(A x, B x), \quad F x=B A x, \quad F x=B^{x} 0
$$

to construct a new function from known functions $A$ and $B$.

Proof. We shall show that $x+y$ can be obtained by substitution alone from $J, K, S$, and the function $2 x=(S S)^{x} 0$. In view of Theorem 1 , this will complete the proof for the case where the initial functions are $S$ and $K$, since we shall be able to compute the sum $A x+B x$ of two given functions.

Notice that

$$
\begin{aligned}
& 2(x+y)^{2}+x+y, \\
& 2(x+y)^{2}+3 x+3 y+1, \\
& 2(x+y)^{2}+5 x+5 y+3, \\
& 2(x+y)^{2}+7 x+7 y+6
\end{aligned}
$$

are four consecutive triangular numbers. Now

$$
4 J(x, y)=2(x+y)^{2}+6 x+2 y
$$

certainly lies between the first and last of these. Hence

$$
K[4 J(x, y)]= \begin{cases}x-3 y-3 & \text { if } x \geqq 3 y+3, \\ 3 x-y-1 & \text { if } x \leqq 3 y+2, y \leqq 3 x-1, \\ 5 x+y & \text { if } y \geqq 3 x .\end{cases}
$$

Thus for $x \geqq 3 y$ we can define both

$$
\begin{aligned}
& x-3 y=K[4 J(x+3, y)], \\
& x+5 y=K[4 J(y, x)] .
\end{aligned}
$$

Hence for $x \geqq y$ we can compute successively

$$
\begin{aligned}
4 x+5 y & =K[4 J(y, 4 x)], \\
x+5 y & =K[4 J(4 x+5 y+3, x)], \\
x-y & =K[4 J(x+5 y+3,2 y)] .
\end{aligned}
$$

Finally, since $J(x, y) \geqq x+y$, we see that $x+y$ may be defined by the formula

$$
x+y=J(x, y)-\{[J(x, y)-x]-y\} .
$$

If the initial functions are $S$ and $L$, we can make a similar argument, using $L[4 J(x, y)]$. 
REMARK. The scheme $F x=A x+B x$ of Theorem 1 was replaced in Theorem 2 by $F x=J(A x, B x)$. Julia Robinson has shown, ${ }^{3}$ by a method similar to that which she used in another paper, ${ }^{4}$ that this scheme may be omitted entirely, if some relatively complicated functions are taken as initial functions.

It is easily seen that the two theorems which we have proved are equivalent to the statement that any of the following pairs of functions could be entered in the last square of the table on p. 929 of PRF: $u+x, K ; u+x, L ; J, K ; J, L$. On the other hand, although the result of Julia Robinson gives a new method of generating primitive recursive functions of one variable, it does not yield an additional entry for this table, which is concerned with the generation of functions of any number of variables. The situation in the two cases is made clear by referring to PRF, $\S 7$, first paragraph, and $\S 8$, second paragraph.

\section{University of CALIFoRNia, BERKELEy}

${ }^{3}$ Julia Robinson, $A$ note on primitive recursive functions, Proc. Amer. Math. Soc. vol. 6 (1955) pp. 667-670.

- Julia Robinson, General recursive functions, Proc. Amer. Math. Soc. vol. 1 (1950) pp. 703-718. See $\S 4$, Theorems 4 and 5. 